\title{
Relating TCR-peptide-MHC affinity to immunogenicity for the design of tumor vaccines
}

\author{
Rachel H. McMahan, ${ }^{1}$ Jennifer A. McWilliams, ${ }^{1}$ Kimberly R. Jordan,, ${ }^{1}$ Steven W. Dow, ${ }^{2}$ \\ Darcy B. Wilson, ${ }^{3}$ and Jill E. Slansky ${ }^{1}$ \\ ${ }^{1}$ Integrated Department of Immunology, University of Colorado at Denver and Health Sciences Center, Denver, Colorado, USA. \\ 2Department of Microbiology, Immunology, and Pathology, Colorado State University, Fort Collins, Colorado, USA. \\ ${ }^{3}$ Torrey Pines Institute for Molecular Studies, San Diego, California, USA.
}

\begin{abstract}
One approach to enhancing the $T$ cell response to tumors is vaccination with mimotopes, mimics of tumor epitopes. While mimotopes can stimulate proliferation of $\mathrm{T}$ cells that recognize tumor-associated antigens (TAAs), this expansion does not always correlate with control of tumor growth. We hypothesized that vaccination with mimotopes of optimal affinity in this interaction will improve antitumor immunity. Using a combinatorial peptide library and a cytotoxic $T$ lymphocyte clone that recognizes a TAA, we identified a panel of mimotopes that, when complexed with MHC, bound the TAA-specific TCR with a range of affinities. As expected, in vitro assays showed that the affinity of the TCR-peptide-MHC (TCR-pMHC) interaction correlated with activity of the $T$ cell clone. However, only vaccination with mimotopes in the intermediate-affinity range elicited functional $T$ cells and provided protection against tumor growth in vivo. Vaccination with mimotopes with the highest-affinity TCR-pMHC interactions elicited TAA-specific $T$ cells to the tumor, but did not control tumor growth at any of the peptide concentrations tested. Further analysis of these $T$ cells showed functional defects in response to the TAA. Thus, stimulation of an antitumor response by mimotopes may be optimal with peptides that increase but do not maximize the affinity of the TCR-pMHC interaction.
\end{abstract}

\section{Introduction}

A seminal goal of immunotherapy is the treatment of cancer with vaccines that elicit potent antitumor immune responses. These vaccines must shift the balance of innate and adaptive immunity from evasion by the tumor to elimination of the tumor (1). Such vaccines must overcome obstacles presented by tumors including the immune suppressive milieu $(2,3)$, cellular heterogeneity (4), and poor reactivity of $\mathrm{T}$ cells for tumor-associated antigens (TAAs). Most identified TAAs are derived from nonmutated proteins produced at high levels by tumor cells (5). As a result, the TCRs of the $\mathrm{T}$ cell repertoire are often of low affinity for these TAAs, due to deletion of T cells with high-affinity TCRs during negative selection in the thymus. Thus, a combination of the weak immunogenicity of TAAs and the tumor environment results in an ineffective antitumor immune response.

These concerns have led to the search for mimotopes (mimics of epitopes, also known as peptides analogs, agonists, heteroclitic peptides, altered peptide ligands, etc.) that enhance the expansion and function of TAA-specific $\mathrm{T}$ cells upon vaccination. This strategy is achieved either by increasing the interaction of the peptide with the restricting MHC through alterations in the anchor residues (6-11) or by selecting peptides that enhance the TCR-peptide-MHC (TCR-pMHC) interaction (12-14). These mimotopes effectively activate TAA-specific $\mathrm{T}$ cells in vitro and

Nonstandard abbreviations used: CT-Ig, TCR covalently linked to $\operatorname{IgG}_{1} ; \mathrm{EC}_{50}, 50 \%$ effective concentration; LANAC, liposome-antigen-nucleic acid complex; $\mathrm{L}^{\mathrm{d}}$-tet, H-2L ${ }^{d}$ tetramer; PMHC, peptide-MHC; RU, resonance unit(s); SPR, surface plasma resonance; TAA, tumor-associated antigen; TIL, tumor-infiltrating lymphocyte. Conflict of interest: The authors have declared that no conflict of interest exists. Citation for this article: J. Clin. Invest. 116:2543-2551 (2006). doi:10.1172/JCI26936 increase TAA-specific T cell expansion in vivo. However, clinical tumor regression does not always correlate with the magnitude of the $\mathrm{T}$ cell responses (15-18).

Thus, effective antitumor immunity may not only depend on the size of the TAA-specific T cell response but also on qualitative or functional aspects of the responding $\mathrm{T}$ cells. Analyses from clinical trials suggest that $T$ cell priming with tumor cells or peptide vaccines may stimulate $T$ cells that cannot mount an effective antitumor response (19-21). For example, tumor-specific circulating T cells from patients with metastatic melanoma lack robust effector functions (22).

The strength of the initial signal received through the TCR - due to antigen concentration $(23,24)$ or the affinity of the stimulating antigen (25-29) - affects the T cell response. Although the affinity must be of sufficient strength to stimulate activation through the TCR, interactions with exceptionally long half-lives results in impaired T cell activation (24, 25, 30-32). These observations imply that the activation of effective TAA-specific T cells may occur only with peptide mimotopes that are within a certain range of affinities.

The experiments described here were designed to determine the optimal binding requirements of mimotopes for effective antitumor immunity. We refer to affinity as the strength of binding of the pMHC to a single TCR molecule and functional avidity as the responsiveness of T cells to peptide antigen (33). The TCR used in this study recognizes the immunodominant $\mathrm{H}-2 \mathrm{~L}^{\mathrm{d}}$-restricted antigen from the transplantable colon tumor, CT26, syngeneic to $\mathrm{BALB} / \mathrm{c}$ mice (34). This epitope is derived from the endogenous retroviral protein gp70, amino acids $423-431$, and is referred to as the $\mathrm{AH} 1$ peptide (35). AH1 peptide binds with relatively high affinity to the $\mathrm{H}-2 \mathrm{~L}^{\mathrm{d}}$ molecule but provides weak protection against CT26 challenge (12). The T cell used was one of $6 \mathrm{~T}$ cell clones generated by limiting dilution in which the TCR sequences were 


\begin{tabular}{|c|c|c|c|c|c|c|c|c|c|}
\hline & \multicolumn{9}{|c|}{ Position } \\
\hline & 1 & 2 & 3 & 4 & 5 & 6 & 7 & 8 & 9 \\
\hline AH1 & $S$ & $P$ & S & $Y$ & V & $Y$ & $\mathrm{H}$ & $Q$ & $\mathrm{~F}$ \\
\hline $\begin{array}{l}\text { Favored amino acid } \\
\text { from library }\end{array}$ & $\begin{array}{c}M \\
E\end{array}$ & $\begin{array}{l}\mathrm{P} \\
\mathrm{N}\end{array}$ & $\begin{array}{l}\mathrm{T} \\
\mathrm{K}\end{array}$ & $\begin{array}{l}Y \\
I\end{array}$ & $\begin{array}{l}\mathrm{T} \\
\mathrm{A} \\
\mathrm{Y}\end{array}$ & $Y$ & $\begin{array}{l}\mathrm{H} \\
\mathrm{W}\end{array}$ & M & $\mathrm{L}$ \\
\hline Mimotope 51 & $E$ & $P$ & $\mathrm{~T}$ & Y & A & $Y$ & $\mathrm{H}$ & M & $\mathrm{L}$ \\
\hline Mimotope 15 & $\mathrm{M}$ & $P$ & K & $Y$ & A & $Y$ & $\mathrm{H}$ & M & $\mathrm{L}$ \\
\hline Mimotope 27 & $M$ & $\mathrm{~N}$ & $\mathrm{~T}$ & $Y$ & $A$ & $Y$ & $\mathrm{H}$ & M & $\mathrm{L}$ \\
\hline Mimotope 39 & $\mathrm{M}$ & $\mathrm{N}$ & K & $Y$ & A & Y & $\mathrm{H}$ & M & $\mathrm{L}$ \\
\hline Mimotope 75 & $\mathrm{E}$ & $\mathrm{N}$ & $\mathrm{T}$ & $Y$ & A & $Y$ & $\mathrm{H}$ & M & $\mathrm{L}$ \\
\hline Mimotope 87 & $\mathrm{E}$ & $\mathrm{N}$ & K & $Y$ & $A$ & $Y$ & $\mathrm{H}$ & M & $\mathrm{L}$ \\
\hline
\end{tabular}

A combinatorial peptide library was screened with a $T$ cell clone that recognizes the $\mathrm{AH} 1 / \mathrm{H}-2 \mathrm{~L}^{\mathrm{d}}$ complex. Those mixtures that consistently induced 2-fold more IFN- $\gamma$ than background from the T cell clone in 3 assays are listed as favored amino acids from the library. Also shown are sequences of 6 mimotope peptides that stimulate a range of cytokine production and binding avidities.

all identical; they all expressed V $\alpha 4.11 / \mathrm{J} \alpha 43$ (AV4S11) and V $\beta 8.3$ / Jß2.6 (BV8S3) gene segments (12). Other investigators have also expanded V 38.3 -expressing clones in response to the AH1 antigen $(36,37)$. The clone lyses CT26 cells in vitro and, when transferred in high concentrations into a mouse bearing a 3-day tumor, eliminates the tumor (35). These results suggest that this clone is an important representative of the repertoire elicited by CT26.

Initial experiments suggested that increasing the affinity of the TCR-pMHC interaction augments tumor protection (12). However, these experiments addressed neither the generality of the correlation nor the range of affinities that produce this response. Using a combinatorial peptide library, we identified a panel of mimotopes with a range of affinities for the AH1-specific TCR. Here we show that all mimotopes with increased affinity for TCR elicited increased numbers of tumor-specific T cells. However, not all mimotopes protect against tumor challenge. pMHC complexes that formed a relatively high-affinity interaction with the TCR elicited T cells that were not functional in vivo and failed to enhance antitumor immunity. The differences we observed between the function of mimotopes in vitro and in vivo have implications for immunotherapy.

\section{Results}

Identification and characterization of peptides using a combinatorial peptide library in positional scanning format. To determine whether increased binding affinity of the TCR-pMHC interaction in the priming of TAA-specific T cells correlates with antitumor immunity, we required a panel of peptides with a range of affinities. To identify these peptides, we screened a combinatorial peptide library (38) with a $\mathrm{T}$ cell clone that recognizes the $\mathrm{AH} 1$ peptide, the immunodominant antigen from the CT26 tumor $(12,35)$. The library included 180 mixtures of peptides of 9 amino acids in which 1 position of the peptides within each mixture harbored a fixed amino acid and 19 different amino acids were possible in each of the remaining 8 positions. $\mathrm{C}$ was omitted from the randomized positions to avoid disulfide bonds but was included in the fixed position. If the $\mathrm{T}$ cell clone consistently produced 2 -fold more IFN- $\gamma$ relative to back- ground when incubated with a peptide mixture, then the constant amino acid from that mixture was defined as a favored amino acid for that position in the peptide (Table 1).

Results from screening the peptide mixtures were used for the design of a panel of peptides that contained all 96 combinations of the favored amino acids (see Table 1). These peptides were synthesized and tested for antigenicity as measured by binding to and activation of the $T$ cell clone. We used 2 methods to determine the relative binding of the mimotope. First, the peptides were incubated with $\mathrm{H}-2 \mathrm{~L}^{\mathrm{d}}$ tetramer $\left(\mathrm{L}^{\mathrm{d}}\right.$-tet $)$ and then used to stain the T cell clone (Supplemental Figure 1A; supplemental material available online with this article; doi:10.1172/JCI26936DS1). Second, the peptides were incubated with T2 cells expressing H-2 $\mathrm{L}^{\mathrm{d}}$ (39) and then stained with TCR covalently linked to IgG $_{1}$ (CT-Ig; ref. 12; Supplemental Figure 1B). These peptides were also cultured with the $\mathrm{T}$ cell clone for 24 hours, and the concentration of IFN- $\gamma$ in the supernatants was determined by ELISA (Supplemental Figure 1C). When complexed with H-2L $\mathrm{L}^{\mathrm{d}}, 35$ of the 96 peptides bound to and induced IFN- $\gamma$ production from the $\mathrm{T}$ cell clone better than the AH1 peptide. I in position $4, \mathrm{~T}$ and $\mathrm{Y}$ in position 5 , and $\mathrm{W}$ in position 7 did not improve binding or IFN- $\gamma$ production. Six peptides were chosen for further analysis because they offered a range of binding in the TCR-pMHC interaction and stimulated varying amounts of IFN- $\gamma$ production from the $\mathrm{T}$ cell clone (Table 1). The amino acid sequences of mimotopes selected from the library were significantly different from the natural AH1 sequence but differed from each other only in the first 3 amino acids. These changes in the $3 \mathrm{~N}$-terminal amino acids were chosen to minimize large structural differences among the mimotopes. However, even single amino acid substitutions may result in structural changes throughout the entire peptide $(40,41)$.

To further characterize the binding of these 6 peptides in the TCR-pMHC interaction, they were incubated with $\mathrm{L}^{\mathrm{d}}$-tet, and the complex was used to stain the $\mathrm{T}$ cell clone that recognizes AH1. As shown in Figure 1A, all of the mimotopes showed increased tetramer binding relative to the AH1 peptide. Mimotopes 15 and 51 bound strongest, followed by mimotopes 39 and 27. Mimotopes 75 and 87 had the weakest tetramer binding of the identified peptides, but still bound the $T$ cell clone stronger than the AH1 peptide. We observed a similar hierarchy of binding when we used soluble multimerized TCRs to stain T2 cells expressing H-2 $\mathrm{L}^{\mathrm{d}}$ bound to peptide (data not shown).

We also determined the affinity of the TCR-pMHC interaction by surface plasma resonance (SPR), which eliminates the potential contribution of binding by the CD8 molecule and activation-induced membrane changes (Figure 1B) $(26,42,43)$. In these experiments soluble TCR was immobilized and the pMHC complexes were in the solution phase (Figure 1B), or soluble TCR was exposed to immobilized $\mathrm{H}-2 \mathrm{~L}^{\mathrm{d}}$ and peptide (data not shown). Monomer $\mathrm{H}-2 \mathrm{~L} /$ mimotope complexes bound to the TCR in the same rank order by SPR analysis as multimer $L^{\mathrm{d}}$-tet/mimotope complexes (tetramers) bound to T cells. Therefore, the $K_{\mathrm{d}}$ values for TCR-pMHC interactions correlate with tetramer binding.

One difference between the peptides that mediate high and intermediate levels of tetramer binding is a $\mathrm{P}$ in position 2. Since position 2 is an anchor position for peptides bound to $H-2 L^{d}(44,45)$, the enhanced tetramer binding could be due to increased peptide binding to the MHC molecules of the tetramer (i.e., better presentation). To test this possibility we compared the pMHC interaction in an $\mathrm{H}-2 \mathrm{~L}^{\mathrm{d}}$ competition assay (Figure $1 \mathrm{C}$ ) (46). The AH1 peptide with 

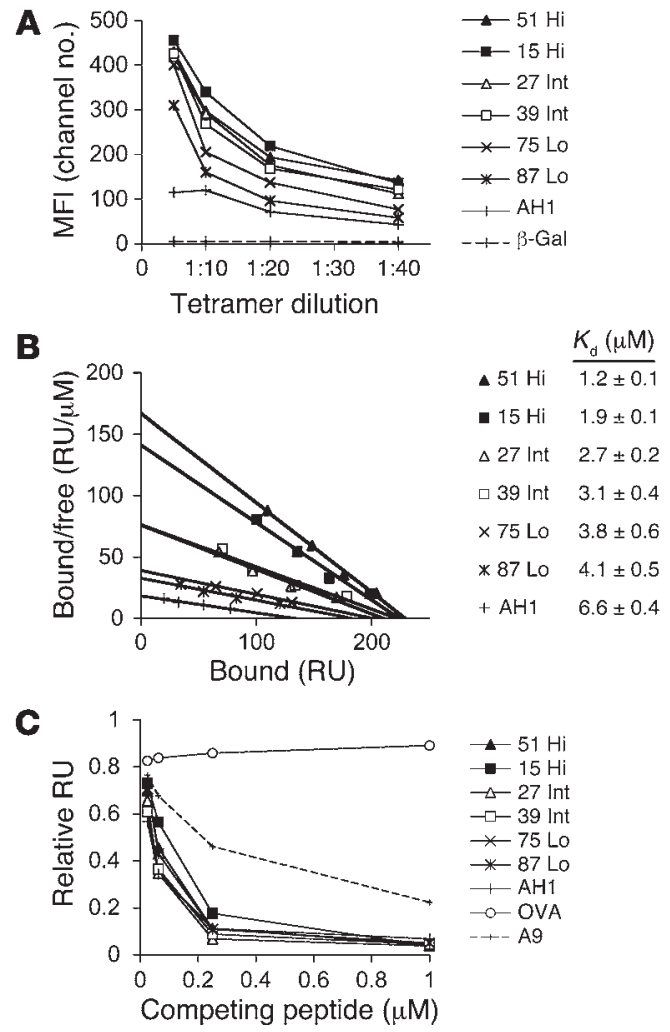

an alanine substitution at position 9 (A9) was shown previously to bind with decreased affinity to $\mathrm{H}-2 \mathrm{~L}^{\mathrm{d}}(12)$, and, as expected, at least 10 -fold more A9 peptide was required to compete for the $\mathrm{H}-2 \mathrm{~L}^{\mathrm{d}}$ relative to the $\mathrm{AH} 1$ peptide. No competition was observed with the $\mathrm{H}-2 \mathrm{~K}^{\mathrm{b}}$-restricted SIINFEKL peptide. All of the mimotopes competed for soluble $\mathrm{H}-2 \mathrm{~L}^{\mathrm{d}}$ as well as the $\mathrm{AH} 1$ peptide, suggesting that the amino acid substitutions did not significantly change binding to MHC. Thus, the enhanced binding mediated by the mimotopes relative to the $\mathrm{AH} 1$ peptide shown in Figure $1 \mathrm{~B}$ is not a result of increased binding to $\mathrm{MHC}$. In addition, the superior binding seen with $\mathrm{H}-2 \mathrm{~L}^{\mathrm{d}}$ bound to mimotopes 15 and 51 cannot be attributed to improved MHC binding since mimotopes 39 and 27 bound MHC with similar if not slightly increased affinity.

Tetramer binding directly correlates with functional activation of the Tcell clone. Tetramer staining does not always correlate with the functional avidity of the TCR-pMHC interaction (47). Therefore, we tested whether the mimotopes stimulate the activation of the $\mathrm{T}$ cell clone and whether the dose response correlates with the binding analyses from Figure 1. We incubated the clone with increasing amounts of peptides and determined production of IFN- $\gamma$, T cell proliferation, and $50 \%$ effective concentration $\left(\mathrm{EC}_{50}\right)$ values, which represent the molar concentration of the peptides that produce $50 \%$ of the maximum proliferation in culture. As shown in Figure 2A, the mimotopes all induced production of IFN- $\gamma$ from the T cell clone at lower concentrations than the AH1 peptide. The low- and intermediateaffinity mimotopes stimulated production of IFN- $\gamma$ with 10 - and 100 -fold less peptide, respectively. The highest-affinity mimotopes, 15 and 51 , stimulated IFN- $\gamma$ production from the clone at the lowest concentration of peptide tested ( $1 \mathrm{pM})$. We observed similar results for proliferation of the clone in response to mimotope stimulation and these results were used to calculate $\mathrm{EC}_{50}$ values. The $\mathrm{EC}_{50}$ values

\section{Figure 1}

Affinity characterization of mimotope peptides. (A) Relative avidities of the TCR-pMHC interactions were determined by staining a T cell clone that recognizes the $\mathrm{AH} 1$ peptide with increasing concentrations of $\mathrm{L}^{\mathrm{d}}$-tet incubated with the indicated peptides. The $\mathrm{H}-2 \mathrm{~L}^{\mathrm{d}}$-restricted $\beta$-gal peptide (64) did not bind the TCR from the T cell clone. Data are presented as mean fluorescence intensity (MFI) and are representative of 3 independent experiments. (B) Affinity binding analysis of the $\mathrm{pMHC}$ complexes for the TCR from the T cell clone were determined using SPR. Increasing concentrations of soluble $\mathrm{H}-2 \mathrm{~L}^{\mathrm{d}}$ monomers incubated with peptide were exposed to the immobilized TCR on a biosensor surface. Equilibrium-binding measurements were used to construct Scatchard plots. $K_{d}$ values were calculated by nonlinear regression analysis of the response units plotted against $\mathrm{H}-2 \mathrm{~L}^{\mathrm{d}}$ concentration (Prism, version 4.0; GraphPad Software). (C) Relative affinities of the peptides for $\mathrm{H}-2 \mathrm{~L}^{d}$ were determined using competition assays. The indicated peptide concentrations and $40 \mu \mathrm{M}$ monomeric $\mathrm{H}-2 \mathrm{~L}^{\mathrm{d}}$ were exposed to the immobilized pMCMV-C4 peptide on a biosensor surface. The amount of $\mathrm{H}-2 \mathrm{~L}^{d}$ bound to PMCMV-C4 is reflected by the change in $\mathrm{RU}$ after 1 minute. Data are presented as $\mathrm{RU}$ relative to binding of empty $\mathrm{H}-2 \mathrm{~L}^{d}$ to the MCMV-C4 peptide. $\mathrm{Hi}$, high affinity; Int, intermediate affinity; Lo, low affinity.

for the high-affinity mimotopes were 3 orders of magnitude higher, the intermediate-affinity mimotopes were 2 orders of magnitude higher, and the low-affinity mimotopes were 2- to 4-fold higher than the $\mathrm{EC}_{50}$ value for the $\mathrm{AH} 1$ peptide. Overall, these data show a correlation between affinity and functional avidity in this system, and major differences in functional avidity result from relatively small changes in the affinity of the TCR-pMHC interaction.

Vaccination with intermediate-affinity peptides provides optimal tumor protection. To determine the correlation between affinity and tumor immunity, mice were primed and boosted with the adjuvant liposome-antigen-nucleic acid complex (LANAC) (48) with 0.1, 1, 10, or $100 \mu \mathrm{g}$ of peptide followed by subcutaneous challenge with the CT26 tumor. As shown in Figure 3, A-D, the AH1 peptide provided no protection against tumor growth at any concentration relative to priming with adjuvant alone (Figure $3 \mathrm{~A}$ ). In addition, minimal tumor protection was observed following vaccination with the high-affinity mimotope 15, which peaked at the 10- $\mu$ g dose (Figure 3C). However, a significant number of mice treated with the intermediate-affinity mimotope 39 remained tumor free for more than 60 days at both $10 \mu \mathrm{g}$ and $100 \mu \mathrm{g}$ (Figure 3, C and D). To determine whether these results represent the other identified mimotopes, we tested the entire panel in this tumor protection assay with $10 \mu \mathrm{g}$ of peptide (Figure 3C). Indeed, vaccination with the low-affinity peptides, 78 and 87 , and the other high-affinity peptide, 51, did not protect against tumor formation. The other intermediate-affinity peptide, 27, protected significant numbers of mice. Thus, unlike what might be predicted from the in vitro experiments, the intermediate-affinity peptides provide superior protection from tumor formation relative to the low- or high-affinity peptides. In addition, vaccination with a range of peptide concentrations demonstrates that the observed differences between the intermediate- and high- 

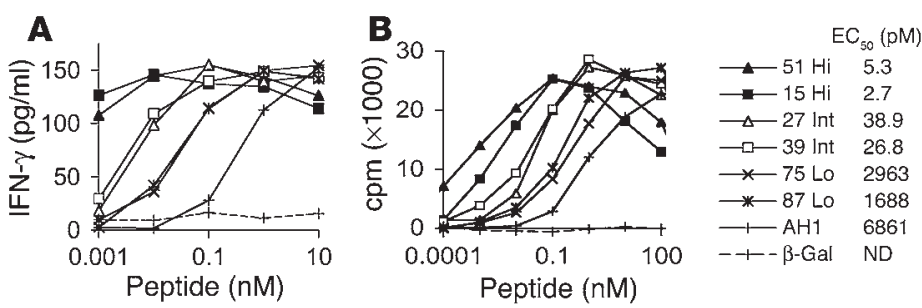

Figure 2

Mimotopes potently activate the T cell clone. The T cell clone was cultured with increasing concentrations of the $\mathrm{AH} 1$ peptide, mimotopes, or the negative control $\beta$-gal peptide. (A) Production of IFN- $\gamma$, as determined by ELISA, from the $T$ cell clone in response to incubation for 24 hours with the indicated peptide. (B) After 24 hours, ${ }^{3} \mathrm{H}$-thymidine was added to the cultures, and ${ }^{3} \mathrm{H}$-thymidine incorporation was measured at 48 hours. Data are representative of 2 experiments per peptide, and each data point was performed in triplicate and averaged. ND, no data ( $\mathrm{EC}_{50}$ was too low to be determined).

affinity peptides are not due to the immunization dose but to an intrinsic response by the responding $T$ cells.

Priming with intermediate-affinity peptides results in optimal AH1-specific Tcell expansion. Although the high-affinity peptides stimulated the $\mathrm{T}$ cell clone better than the intermediate-affinity peptides in vitro, they did not protect mice from tumor challenge. To determine whether vaccination with the high-affinity mimotopes, 15 and 51, stimulate the clonal expansion of tumor-specific $T$ cells, mice were injected with CT26 tumor cells, palpable tumors were established, and then mice were vaccinated with the peptides and adjuvant. To ensure that the tumor was not cured by the vaccine (so that tumor-infiltrating lymphocytes [TILs] could be obtained), 2-fold more tumor cells were transplanted relative to the protection assay in Figure 3. TILs were isolated and stained with $\mathrm{L}^{\mathrm{d}}$-tet/AH1 on day 14 (Figure 4A). All tumors from mice vaccinated with the mimotopes harbored an increased frequency of AH1-specific T cells relative to unvaccinated tumor-bearing mice or mice vaccinated with the AH1 peptide (Figure $4 \mathrm{~B}$ ). These data suggest that the lack of tumor protection pro- vided by the high-affinity peptides is not due to an absence of AH1-specific T cells at the tumor site.

To determine whether activation phenotype contributes to functional differences, we examined the activation markers and TCR expression of AH1-specific T cells in mice primed with the AH1 peptide and both intermediate- and high-affinity mimotopes. TILs were stained with antibodies to the activation markers CD69, CD44, and CD122 and with an antibody against the $\beta$ chain of the TCR (Figure 4C). Tetramer-positive $\mathrm{T}$ cells from mice primed with mimotope 39 (intermediate affinity) and mimotope 15 (high affinity) were similarly positive for the early activation marker CD69 and the T cell memory marker CD122 (IL-2R $\beta$ ) relative to cells primed with the AH1 peptide. Expression of CD44, an adhesion molecule and marker for effector T cells, was increased on TILs from the mimotope-primed mice relative to TILs from the mice primed with the AH1 peptide. However, there was no consistent difference in CD44 expression on TILs from mice primed with the 39 and 15 mimotopes, although in some experiments $\mathrm{T}$ cells from mice vaccinated with mimotope 39 had higher levels than those vaccinated with mimotope 51 (data not shown). Finally, there was no difference in cell surface expression of TCR on the T cells elicited by AH1 or mimotopes. This analysis demonstrates that the $\mathrm{T}$ cells responding to both the high- and intermediate-affinity vaccines are similarly antigen experienced, as measured by activation marker expression. Interestingly, the TILs from AH1-vaccinated mice appeared to be less activated, despite being recruited to tumor.

$T$ cells elicited by high-affinity peptides have defects in effector function. A number of studies investigating $\mathrm{T}$ cells that respond to tumors show defects in one or more effector functions (e.g., cytotoxicity and cytokine production; refs. 1, 22, 49). Rubio et al. showed that in human cancer patients most cytotoxic TAA-specific $\mathrm{T}$ cells express the protein LAMP-1 (CD107a), a marker for lysosomal granule exocytosis (19). In addition, CD107a expression correlates with cytotoxicity in a viral antigen model in mice (50). Thus, we examined the expression CD107a on mimotope- or AH1-primed T cells in response to the AH1 peptide. We harvested TILs from mimo-

\section{Figure 3}

Vaccination with mimotopes of intermediate affinity most effectively protects against tumor challenge. $\mathrm{BALB} / \mathrm{c}$ mice were primed on days -17 and -10 with (A) 0 or $0.1 \mu \mathrm{g}$, (B) $1 \mu \mathrm{g}$, (C) $10 \mu \mathrm{g}$, or (D) $100 \mu \mathrm{g}$ peptides and LANAC (adjuvant) or LANAC alone followed by injection of $5 \times 10^{4}$ CT2 6 tumor cells on day 0 (subcutaneous, back left flank). Tumorfree survival was monitored and plotted using the Kaplan-Meier method (Prism, version 4.0; GraphPad Software). Survival among groups was compared using the log-rank test (Prism, version 4.0; GraphPad Software). ${ }^{*} P<0.004,{ }^{* \star} P<0.006$ versus mimotope 15. All vaccination groups consisted of 8 mice per peptide with the exception of the $10 \mu \mathrm{g}$ dose, for which 16 mice per peptide were challenged (except for the mimotope 27, 75, and 87 groups, which each consisted of 12 mice).
A

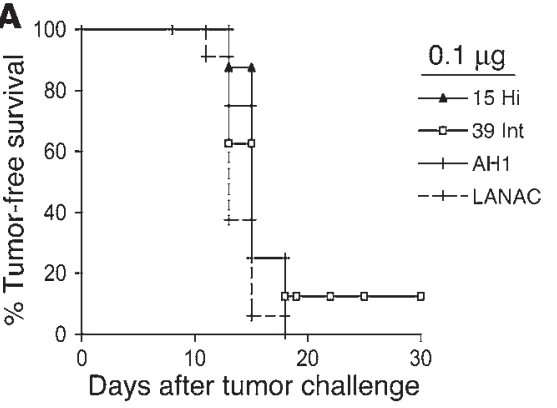

C

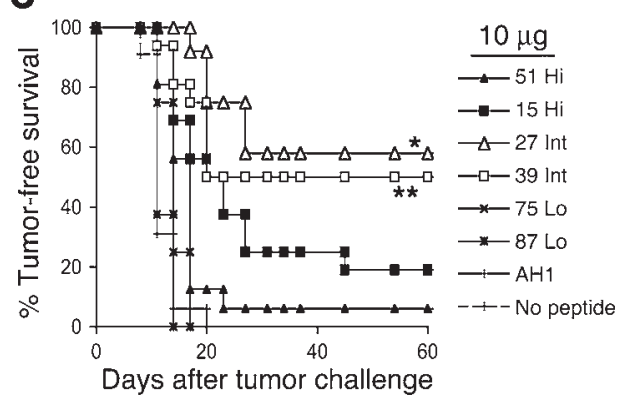

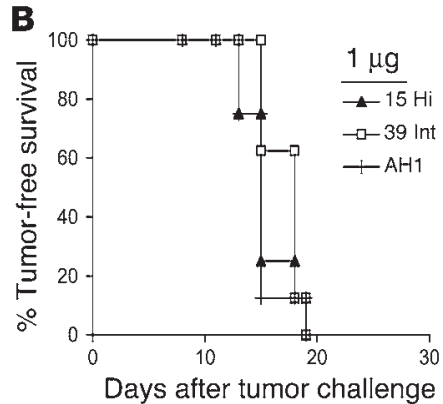

D

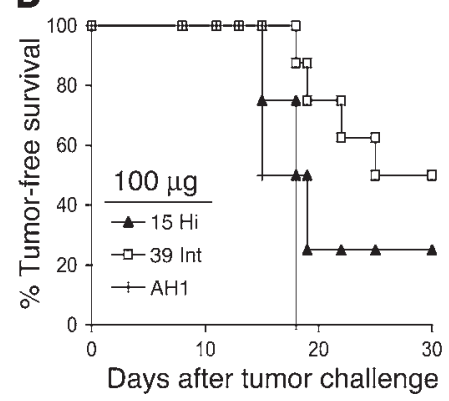


A
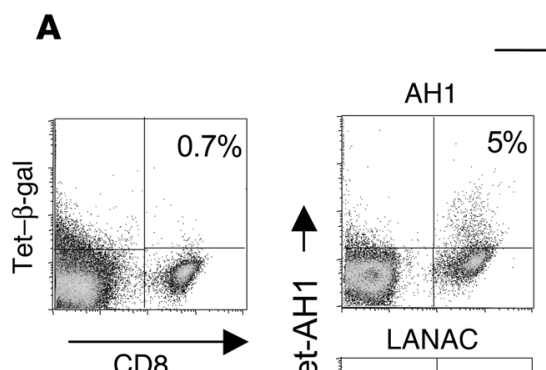

Increasing affinity

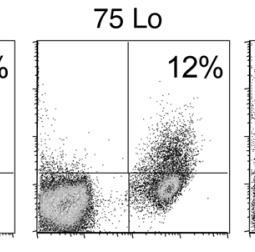

87 Lo
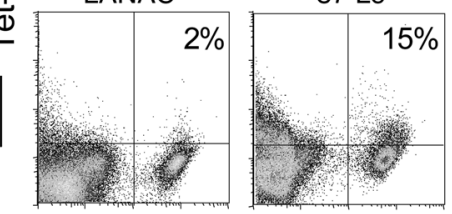

CD8

B

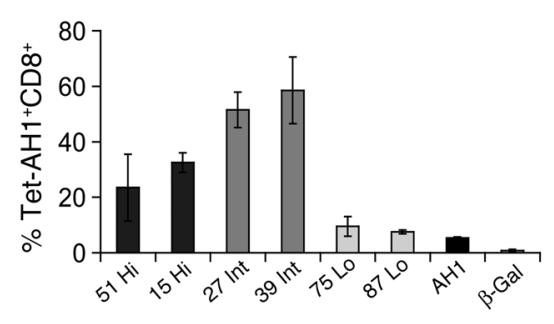

C

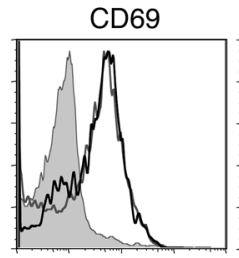

CD122

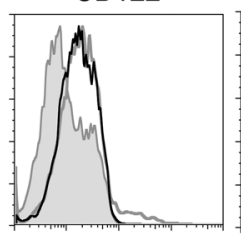

$15 \mathrm{Hi}$

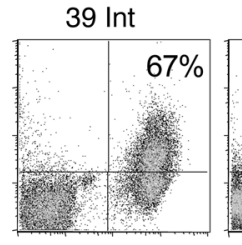

27 Int

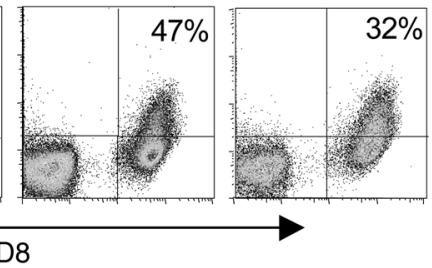

CD44

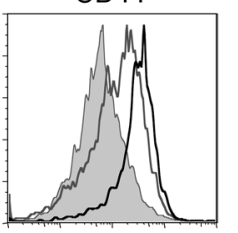

$\operatorname{TCR} \beta$

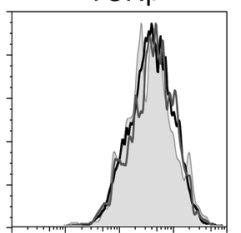

Figure 4

The AH1-specific $\mathrm{T}$ cell response was strongest after priming with the intermediate-binding mimotopes. (A) BALB/c mice with day 4 established tumors were injected with LANAC and the indicated peptides. On day 14, TILs were isolated and stained ex vivo with CD8 antibody and $L^{d}$-tet bound to either the AH1 or $\beta$-gal peptide. The percentages of tetramer-positive CD8 ${ }^{+}$cells are indicated. (B) Using the methods from A, the mean \pm SEM of $\mathrm{AH} 1$-specific $\mathrm{CD}^{+}$ TILs was calculated for 4 mice per vaccine. (C) Ld-tet/AH1-positive CD8 ${ }^{+} \mathrm{T}$ cells from tumors of mice vaccinated with $\mathrm{AH} 1$ (filled), mimotope 39 (black line), or mimotope 15 (gray line) peptides were stained ex vivo with CD69, CD44, CD122, and TCR $\beta$ antibodies. Staining of activation markers was not performed for the other mimotopes. tope- or AH1-primed mice and compared their CD107a expression following a 5-hour incubation with the AH1 peptide ex vivo. There was no significant difference in CD107a expression on T cells from mice vaccinated with the AH1 peptide or mimotopes, indicating that the defects following vaccination with high-affinity mimotopes are not in this part of the cytolytic pathway (Figure 5A).

The importance of IFN- $\gamma$ in an effective antitumor $T$ cell response has been shown previously $(1,51)$. Thus, we tested TILs from vaccinated mice for the production of IFN- $\gamma$ in response to the AH1 peptide. TILs from mice vaccinated with low- and intermediate-affinity mimotopes produced IFN- $\gamma$ in response to the AH1 peptide (Figure $5 \mathrm{~B}$ ). In contrast, TILs from mice vaccinated with the high-affinity mimotopes produced significantly less IFN- $\gamma$ following stimulation with the AH1 peptide (Figure 5B). The absence of IFN- $\gamma$ production following ex vivo peptide stimulation was observed over a range of peptide concentrations (Figure 5C). Furthermore, ex vivo stimulation with the high-affinity mimotope peptides used in the vaccination did not induce cytokine production (Figure 5D). This lack of cytokine production was not due to downregulation of the TCR since cell surface staining for the TCR $\beta$ was the same for TILs from mimotope 39- and mimotope 15-vaccinated mice. Furthermore, T cells elicited by the high-affinity peptides are not completely unresponsive, since ex vivo stimulation with PMA and ionomycin induced IFN- $\gamma$ production at levels similar to those primed with intermediate-affinity mimotopes (data not shown). Taken together, these data suggest that vaccination with peptides of high affinity leads to the expansion of TAA-specific T cells that lack the ability to produce IFN- $\gamma$, likely contributing to their inferior tumor protection.

\section{Discussion}

The notion that TCRs are often of low affinity for TAA and not sufficiently activated by TAA has led to the hypothesis that antitumor immunity can be generated by activating TAA-specific $T$ cells using mimotopes of higher affinity. Using a combinatorial peptide library we identified a panel of peptide mimotopes for the T cell epitope AH1 from the transplantable CT26 tumor (Table 1). We studied 6 mimotopes that, when complexed with $\mathrm{H}-2 \mathrm{~L}^{\mathrm{d}}$, have increasing affinities for a TCR that recognizes the H-2Ld/AH1 complex. These mimotopes all showed similar binding to $\mathrm{H}-2 \mathrm{~L}^{\mathrm{d}}$, suggesting that the increase in affinity of the TCR-pMHC interaction is a result of enhanced binding of the MHC-mimotope to the TCR. Using these mimotopes, we found that to induce an optimal antitumor response, the affinity of the TCR-pMHC interaction must be higher than the TAA, but cannot be too high. Thus, the intermediate-affinity peptides elicited optimal antitumor responses.

The sequence of these mimotopes differs in the first 3 of 9 amino acids. The second amino acid is an anchor residue for binding the $\mathrm{H}-2 \mathrm{~L}^{\mathrm{d}}$ molecule $(44,45)$. The high-affinity mimotopes and the AH1 peptide have the consensus $\mathrm{P}$ at this position. Typically mimotopes are designed with consensus anchor residues to ensure optimal presentation. Although the intermediate-affinity mimotopes, 39 and 27, have $\mathrm{N}$ at this position, $\mathrm{N}$ has been identified in other H-2L ${ }^{\mathrm{d}}$-restricted peptides (Tum QNHRALDL; ref. 52). Using the SPR competition assay in Figure 1C, we did not detect differences in binding $\mathrm{H}-2 \mathrm{~L}^{\mathrm{d}}$ molecules whether position 2 is a $\mathrm{P}$ or an $\mathrm{N}$, but it is interesting to note that the peptides that were most protective did not have the consensus anchor residue at position 2 . 

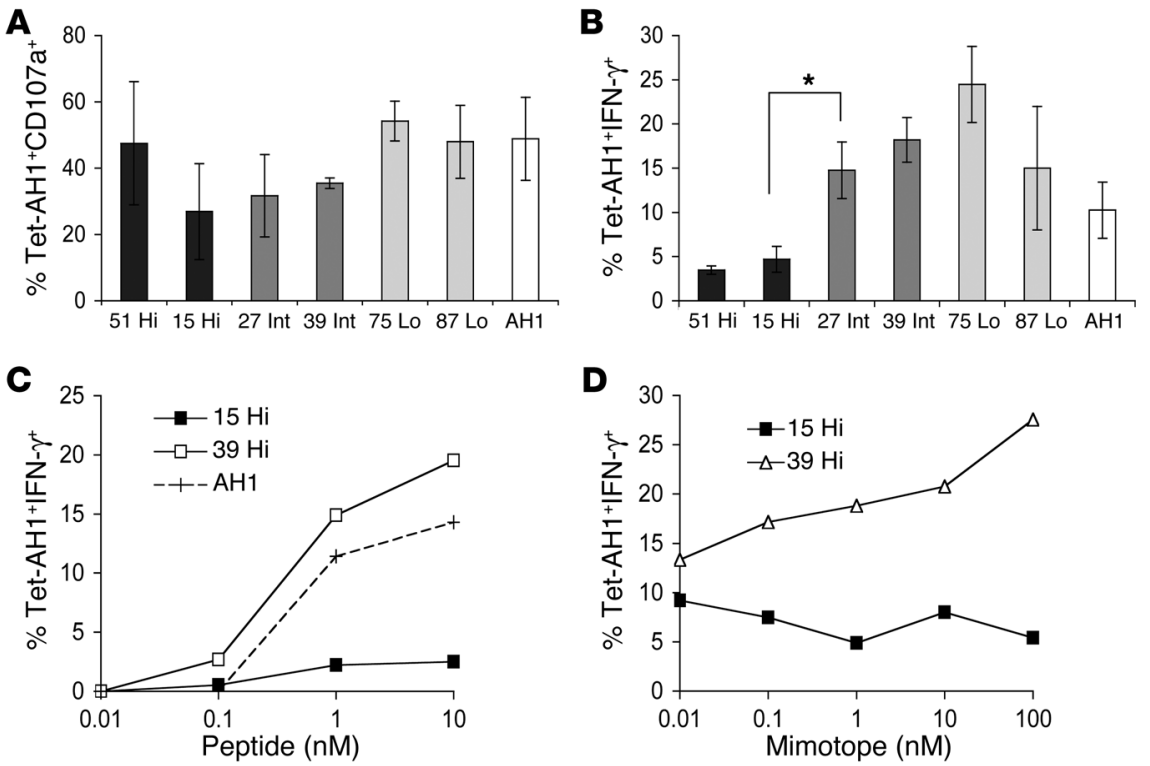

Figure 5

TILs elicited by high-affinity mimotopes produce cytotoxic granules, but have impaired production of IFN- $\gamma$ in response to antigen. BALB/c mice with day 4 established CT26 tumors were primed with LANAC alone or LANAC with the indicated peptide on days 4 and 6. (A and B) CD8+ TILs were isolated on day 14 and stimulated with $1 \mu \mathrm{M} \mathrm{AH} 1$ peptide for 5 hours at $37^{\circ} \mathrm{C}$. Cells were stained with CD8 antibody and $\mathrm{L}^{\mathrm{d}}$-tet/AH1 as well as (A) CD107a, a marker of cytotoxic granules, or (B) IFN- $\gamma$ antibody. The mean \pm SEM was calculated for 4 mice per peptide. ${ }^{\star} P=0.0125$. Functional analysis of TILs from mice vaccinated with LANAC alone was not possible due to low numbers of $\mathrm{AH} 1$-specific T cells. (C and $\mathbf{D}) \mathrm{CD}^{+}$TILs isolated from mice vaccinated with $\mathrm{AH} 1$, mimotope 39 , or mimotope 15 were stimulated with graded concentrations of $(\mathbf{C})$ the $\mathrm{AH} 1$ peptide or (D) the mimotope used for vaccination and stained for intracellular IFN- $\gamma$ after 5 hours at $37^{\circ} \mathrm{C}$.

Increasing the affinity of the TCR-pMHC interaction correlated with increased proliferation and IFN- $\gamma$ production from the $\mathrm{T}$ cell clone in vitro (Figure 2). However, the increased binding did not correlate with antitumor immunity in vivo; vaccination with mimotopes whose $\mathrm{EC}_{50}$ was 3 orders of magnitude higher than the TAA resulted in no increase in antitumor immunity (Figure 3 ). Mimotopes that promote antitumor immunity had a midrange affinity that correlated with an $\mathrm{EC}_{50} 2$ orders of magnitude higher than the wild-type TAA. Interestingly, a previously characterized mimotope for the AH1 peptide, A5 (12), which protects mice from tumor challenge, also falls within the range of the effective intermediate-affinity peptides (data not shown). It should be noted that none of the mimotopes chosen from the library exist in the mouse genome so the lack of antitumor activity seen by the high-affinity mimotopes cannot be attributed directly to central tolerance.

One important question that arises from these experiments is how representative results from one $\mathrm{T}$ cell clone are relative to a responding population of $\mathrm{T}$ cells. As discussed in the Introduction, the TCR used was identified multiple times, and the clone it was derived from had low functional avidity for its cognate ligand, as expected for T cells specific for self antigens (Figure 2). Thus, both the precursor frequency and functional avidity likely represent the responding population. However, future experiments to identify mimotopes using nonclonal $\mathrm{T}$ cell populations that respond to the TAA will address whether a clone will predict optimal mimotopes for a polyclonal $\mathrm{T}$ cell population.
Increasing evidence suggests that the quality, not quantity, of the responding $\mathrm{T}$ cells determines the efficacy of the response. The mimotopes in the loweraffinity range expanded fewer AH1-specific $\mathrm{T}$ cells, potentially contributing to their inability to protect against tumor challenge (Figure 4A). Vaccination with the intermediate-affinity mimotopes results in more AH1-specific T cells relative to the highest-affinity mimotopes. TILs from mice vaccinated with both intermediateand high-affinity mimotopes were antigen experienced and displayed CD107a in response to $\mathrm{AH} 1$ peptide (Figure 5).

A more striking difference between $\mathrm{T}$ cells expanded by the intermediate- and high-affinity mimotopes was the observed functional differences. TILs from mice vaccinated with the high-affinity mimotopes produced significantly less IFN- $\gamma$. A similar loss of antigen-specific IFN- $\gamma$ production by TILs was recently reported in melanoma patients (49). IFN- $\gamma$ is a potent activator of macrophages and increases the expression of MHC class I on tumor cells, making it crucial for an effective response against solid tumors $(1,51,53)$. The inability of the TAA-specific T cells to produce IFN- $\gamma$ in response to vaccination with high-affinity mimotopes may explain why large numbers of $T$ cells do not protect against tumor growth. It is important to note that although IFN- $\gamma$ production was impaired following stimulation with the AH1 or mimotope peptide, treatment with PMA and ionomycin induced cytokine production, arguing against a global defect in these cells. High-affinity TCR-pMHC interactions have been shown to induce $T$ cell deletion in the periphery $(30,54)$. High-affinity mimotopes may lead to deletion of the highest-avidity $\mathrm{T}$ cells and/or an expansion of a lower-avidity population of $\mathrm{T}$ cells. These low-avidity $\mathrm{T}$ cells have a low sensitivity for peptide and require high concentrations of cognate peptide for both cytotoxic activity and cytokine production $(20,21)$. This rationale predicts that vaccination with mimotopes of different affinities results in the expansion of $\mathrm{T}$ cells of varying avidity for the AH1 peptide. Therefore, the intermediate-affinity mimotopes may elicit a different repertoire of $\mathrm{T}$ cells relative to those expanded by the high-affinity mimotopes, resulting in improved antitumor activity. Although preliminary analysis of the responding repertoires of $T$ cells elicited by both intermediate- and high-affinity mimotopes revealed no obvious differences in TCR V $\beta$ chain expression (data not shown), critical differences in the CDR3 sequence may exist.

Alternatively, strong stimulation through the TCR by the highaffinity peptides may lead to the expansion of functionally anergic $T$ cells that do not respond to further stimulation through the TCR. This response prevents autoimmunity following activation of self-reactive $\mathrm{T}$ cells in the periphery and occurs in mice harboring large numbers of transgenic $\mathrm{T}$ cells reactive against self antigens (55-57). Similar to what we observed, these autoantigenspecific T cells proliferate and upregulate CD44 and CD69, but 
fail to develop effector functions. Functional impairment of CD8 $\mathrm{T}$ cells is also observed following lymphocytic choriomeningitis virus infection, where chronic viral loads lead to an exhausted population of $\mathrm{T}$ cells that lose the ability to produce IFN- $\gamma$ (58, 59). In addition, administration of high-affinity peptide analogs of a myelin basic protein peptide prevent the onset of experimental autoimmune encephalomyelitis, suggesting that vaccination with mimotopes above an optimal affinity may be detrimental in inducing an optimal $\mathrm{T}$ cell response (54). Further analysis of these functionally unresponsive cells is necessary to understand the mechanisms of functional impairment.

There is an affinity threshold for peptide vaccines below which $\mathrm{T}$ cells will not proliferate, and this threshold can be overcome by vaccination with peptides that increase TCR-pMHC affinity (6-10, $12-14)$. We propose here that there is also an upper boundary for the affinity of peptide vaccines above which the expansion of functionally unresponsive T cells occurs. MHC-mimotope complexes with intermediate affinity for the TCR induced the expansion of TAA-specific $T$ cells that produce IFN- $\gamma$, demonstrating that this state of unresponsiveness was not evoked with lower-affinity antigen stimulation. These data have important implications for the design of peptide vaccines, since much of the previous work with mimotopes has used functional stimulation of a $\mathrm{T}$ cell clone as a readout for vaccine effectiveness.

In summary, we show here that while peptide stimulation of the $\mathrm{T}$ cell clone in culture correlates well with physical binding parameters involving the TCR-pMHC complex, it does not correlate well with immunogenicity of the same peptide against the tumor. The peptides of highest affinity may not be appropriate in the design of effective antitumor vaccines; as immunogens, these high-affinity mimotopes may functionally inactivate the relevant responding $\mathrm{T}$ cells by expanding cells with defective cytokine profiles.

\section{Methods}

Peptides. All large-scale peptide syntheses were purified to greater than $95 \%$ by the manufacturers. The sequences of the peptide mimotopes are shown in Table 1. The sequences of the other peptides used are TPHPARIGL ( $\beta$-gal), SPSYVYHQA (A9), SIINFEKL $\left(\mathrm{OVA}_{257-264}\right)$ and YPHCMPTNL (pMCMV-C4); the A and C substitutions for which the A9 and PMCMVC4 peptides, respectively, are named are shown in bold.

Cell culture. The CT26 colon carcinoma cell line was cultured as described previously (12). The $\mathrm{T}$ cell clone that recognizes the AH1 peptide was generated from the spleen of a BALB/c mouse primed with irradiated CT26-GM and was cultured as described previously (12). Briefly, $2 \times 10^{6} \mathrm{~T}$ cells were cultured in complete medium and stimulated every 7 days with $1 \times 10^{6}$ splenocytes, $10 \mathrm{U} / \mathrm{ml} \mathrm{IL-2,} \mathrm{and} 10 \mu \mathrm{g} / \mathrm{ml}$ peptide.

Peptide library in positional scanning format. An N-terminal nonacetylated C-terminal amide L-amino acid nonapeptide library (PCL97-3) was prepared in a positional scanning format at Multiple Peptide Systems as described previously (38). Assays to determine which peptide mixtures stimulate cytokine production from the $\mathrm{T}$ cell clone were performed in 96-well round-bottomed plates. Clonal T cells $\left(2 \times 10^{4}\right)$, irradiated BALB/c spleen cells $\left(2 \times 10^{4}\right)$, human $\beta_{2} \mathrm{~m}(2.5 \mu \mathrm{g} / \mathrm{ml})$, IL-2 $(12.5$ $\mathrm{U} / \mathrm{ml})$, and peptide library mixtures $(100 \mu \mathrm{g} / \mathrm{ml})$ were cultured in complete medium. Supernatants from the cultures were analyzed in 3 separate assays by ELISA for IFN- $\gamma$ after 24 hours per the manufacturer's instructions (Pierce Biotechnology; Endogen). Small-scale synthesis of 96 individual peptides was done on an automated synthesizer by the simultaneous multiple peptide synthesis method (Spyder Instruments Inc.) and not further purified.
Antibodies and tetramers. CD8-cychrome, TCR $\beta$-FITC, CD44-allophycocyanin (CD44-APC), CD69-FITC, and CD122-FITC antibodies were purchased from BD Biosciences - Pharmingen. IFN- $\gamma$-APC and CD107a-FITC antibodies were purchased from eBioscience. $\mathrm{H}-2 \mathrm{~L}^{\mathrm{d}}$ multimers ( $\mathrm{L}^{\mathrm{d}}$-tet) were produced in a baculovirus expression system as previously described (60). Briefly, high 5 (Hi5) insect cell cultures were infected with baculovirus expressing an $\mathrm{H}-2 \mathrm{~L}^{\mathrm{d}}$-BirA fusion protein from the polyhedron promoter and $a \beta_{2} \mathrm{~m}$ protein from the $\mathrm{P} 10$ promoter. Soluble protein was enriched on a 28.14.8 antibody (anti-H-2 $\mathrm{L}^{\mathrm{d}}$ ) column. The BirA sequence was biotinylated per the manufacturer's instructions (Avidity), and $\mathrm{H}-2 \mathrm{~L}^{\mathrm{d}}$-biotin was then combined at a 1:8 ratio with streptavidin-PE (BioSource International). The resulting complexes were purified on a Sephadex 200 column. Purified $\mathrm{L}^{\mathrm{d}}$-tet was incubated with approximately 200 -fold excess peptide for 24 hours prior to staining. Background tetramer staining was determined with $L^{\mathrm{d}}$-tet incubated with the $\mathrm{H}-2 \mathrm{~L}^{\mathrm{d}}$-restricted $\beta$-gal peptide.

Proteins. The soluble double-chain TCR was subcloned into a baculovirus expression system and purified as previously described (61). Briefly, the V $\beta 8.3 / \mathrm{J} \beta 2.6$ chain was amplified from the CT-Ig expression plasmid (12) with primers 5'-ATGGAGGCTGCAGTCACCCAAAGC-3' and 5'-CTGGTACACAGCAGGTTCCGGATTCTGGATGT-3' to introduce PstI and $B g l I I$ sites. The fragment was inserted into a modified baculovirus transfer vector downstream of the polyhedron promoter and the V $\beta 8.2$ leader sequence. The V $\alpha 4.11 / \mathrm{J} \alpha 43$ chain was amplified using primers 5 '-CTAACAGGGAACGTCTGAGCT-3' and 5'-TGCTGGGTCCGCGGTGACTCAGTAACCCAGAT- $3^{\prime}$, then with the same forward primer and 5'-GGATTCTGTGGGTGCAGATTTGCTGGGTCCGCGGT-3' to introduce the AvrII and SacI sites. The fragment was inserted into the baculovirus transfer vector downstream of the P10 promoter and the Vo11 leader sequence. The sequence was confirmed, and the transfer vector was cotransfected with BaculoGold viral DNA (BD Biosciences - Pharmingen) into Sf9 insect cells. Protein conformation was confirmed by ELISA and large-scale protein production was carried out in $\mathrm{Hi} 5$ insect cells. Protein was enriched from supernatants by affinity chromatography using a HAM-597 antibody (anti-TCR C $\beta$ ) column followed by a Sephadex 200 column. Soluble monomeric $\mathrm{H}-2 \mathrm{~L}^{\mathrm{d}}$ was similarly produced, but the BirA sequence from the $\mathrm{L}^{\mathrm{d}}$-tet construct was replaced with a fragment encoding a stop codon.

Divalent forms of the TCR (CT-Ig) and H-2 $\mathrm{L}^{\mathrm{d}}\left(\mathrm{L}^{\mathrm{d}}-\mathrm{Ig}\right)$ multimerized with $\mathrm{IgG}_{1}$ were purified as described previously (12).

Biosensor analysis. We performed real-time SPR experiments using a Biacore 2000 instrument (Biacore). All immobilization and binding experiments were performed at $25^{\circ} \mathrm{C}$ at a flow rate of $5 \mu \mathrm{l} / \mathrm{min}$. Solution phase ligands were diluted in HBST (10 mM HEPES, pH 7.5; 3.4 mM EDTA; 15 mM sodium chloride; $0.1 \%$ Tween 20 ). The $\mathrm{H}-2 \mathrm{~L}^{\mathrm{d}}$ peptide competition assays were performed as previously described $(12,46)$. Briefly, between 100 and 200 resonance units (RU) of the $\mathrm{H}-2 \mathrm{~L}^{\mathrm{d}}$-restricted $\mathrm{pMCMV}-\mathrm{C} 4$ peptide were coupled to a Sensor Chip CM5 (Biacore). Monomeric H-2 $\mathrm{L}^{\mathrm{d}}$ and decreasing amounts of competing peptide $(1,0.25,0.125$, and $0.0625 \mu \mathrm{M})$ were exposed to the pMCMV-C4 peptide at $5 \mu \mathrm{l} / \mathrm{min}$ for 1 minute. After each binding reaction, the surface was regenerated with $50 \mathrm{mM}$ phosphoric acid.

For the TCR-pMHC affinity measurements approximately 5,000 RU of the ADO-304 anti-TCR antibody (gift from J. Kappler, National Jewish Medical and Research Center, Denver, Colorado, USA) in $25 \mathrm{mM}$ sodium acetate ( $\mathrm{pH}$ 5.1) was coupled to the Sensor Chip CM5 with amine chemistry (NHS/EDC) as determined by the BIAevaluation 3.0 Application Wizard (Biacore). Soluble TCR $(10 \mu \mathrm{g} / \mathrm{ml})$ was then bound to the immobilized antibody at a flow rate of $5 \mu \mathrm{l} / \mathrm{min}$ for 1 minute. The chip surface was cleared of excess protein with a 5 -minute wash with $\mathrm{HBST}$ and monomeric $\mathrm{H}-2 \mathrm{~L}$ and peptide $(1.25,2.5,5$, or $10 \mu \mathrm{M})$ was exposed to the chip at $5 \mu \mathrm{l} / \mathrm{min}$. Background refractive index shift was subtracted as determined by a signal from a flow cell prepared by the same method without TCR protein. 
IFN- $\gamma$ ELISA and proliferation assays. Stimulation of the T cell clone was performed by incubating $2 \times 10^{4} \mathrm{~T}$ cell clones with peptide, $1 \times 10^{5}$ splenocytes and $0.5 \mathrm{U} / \mathrm{ml} \mathrm{IL-2}$ for 24 hours at $37^{\circ} \mathrm{C}$ in a 96 -well plate. Supernatants were harvested, and detection of IFN- $\gamma$ production from the $\mathrm{T}$ cell clone was assayed by ELISA per the manufacturer's instructions (Pierce Biotechnology; Endogen). For proliferation assays $1 \mu \mathrm{Ci}$ of ${ }^{3} \mathrm{H}$-thymidine was added to the wells at 24 hours. Cells were harvested at 48 hours, and thymidine incorporation was measured using a beta scintillation counter. Results shown are representative of 2 experiments per peptide, and each data point was performed in triplicate and averaged. $\mathrm{EC}_{50}$ values were calculated using Prism software (version 4.0; GraphPad Software).

Mice and immunizations. Six- to eight-week-old female BALB/cAnNCr mice were purchased from the National Cancer Institute/Charles River Laboratories. All experimental protocols were reviewed and approved by the Institutional Animal Care and Use Committee of National Jewish Medical and Research Center (Denver, Colorado, USA). For tumor protection assays, mice were primed (day -17) and boosted (day -10) intraperitoneally with LANAC alone or LANAC and peptide. On day 0 , mice were injected subcutaneously in the left back flank with $5 \times 10^{4} \mathrm{CT} 26$ tumor cells (34). Tumor-free survival was assessed by palpation at the site of injection. Tumors developed in most untreated mice within 12-14 days. The statistics were analyzed using Prism 4.0 software (GraphPad Software).

For analysis of TILs, mice were injected subcutaneously with $1 \times 10^{5} \mathrm{CT} 26$ cells. On days 4 and 6 , mice were injected with LANAC alone or LANAC and peptide and tumors were harvested on day 14. Tumors were disrupted into a single-cell suspension through a metal screen and stained.

Preparation of LANAC vaccines. LANACs are composed of cationic liposomes, plasmid DNA, and peptide antigens. Briefly, equimolor concentrations of DOTIM (octadecenolyoxy\{ethyl-2-heptadecenyl-3 hydroxyethyl\} imidazolinium chloride; Sigma-Aldrich) and cholesterol (Avanti Polar Lipids Inc.) were dissolved in chloroform in glass tubes to a final concentration of $2 \mathrm{mM}$. The solution was dried to a film in a vacuum desiccator. The lipids were rehydrated in $5 \%$ dextrose in water at $50^{\circ} \mathrm{C}$ for 50 minutes and incubated for 2 hours at room temperature. The solution was processed and mixed with plasmid DNA as previously described $(62,63)$. The peptides were gently mixed with the liposome-DNA complexes at the concentrations indicated in Results and the figure legends prior to injection.

Flow cytometric analysis. To examine specific ligand binding, $5 \times 10^{5} \mathrm{~T}$ cell clones were incubated on ice for 1 hour with $\mathrm{L}^{\mathrm{d}}$-tet that was preincubated with
$1 \mathrm{mM}$ peptide. For staining with CT-Ig, $3 \times 10^{5} \mathrm{~T} 2$ - $\mathrm{L}^{\mathrm{d}}$ cells were incubated overnight at $25^{\circ} \mathrm{C}$ in serum-free medium and then incubated with $10 \mu \mathrm{M}$ peptide for 2 hours at $37^{\circ} \mathrm{C}$. CT-Ig was added, and the cells were incubated on ice for 2 hours. CT-Ig binding was visualized with IgG $_{1}$-Biotin (BD Biosciences) and avidin-PE (BioSource International). TILs were stained with the Fc antibody 2.4G2, $\mathrm{L}^{\mathrm{d}}$-tet, and antibodies to cell surface markers. Intracellular IFN- $\gamma$ staining was performed with BD Cytofix/Cytoperm kit (BD Biosciences) per the manufacturer's instructions. Briefly, $5 \times 10^{6}$ cells were incubated for 5 hours at $37^{\circ} \mathrm{C}$ with $\mathrm{AH} 1$, mimotope, or the negative control $\beta$-gal peptide in the presence of Brefeldin A. Positive control wells were activated with $100 \mathrm{nM}$ PMA and $500 \mathrm{nM}$ ionomyocin for 4 hours. For CD107a staining, cells were stimulated by a similar method in the presence of $0.5 \mu \mathrm{g}$ CD107a antibody.

Statistics. Data are presented as mean \pm SEM. Statistical differences between 2 groups were analyzed using an unpaired 2-tailed Student's $t$ test. Survival data were analyzed by Kaplan-Meier survival curves and log-rank statistics. A $P$ value less than 0.05 was considered to be statistically significant.

\section{Acknowledgments}

We thank Miguel T. Gonzalez for technical support. We are grateful to Ross Kedl for supplying baculovirus reagents for $\mathrm{L}^{\mathrm{d}}$-tet production, Terry Potter for the 28.14.8 antibody, and John Kappler and Philippa Marrack for advice and protein production reagents. This work was supported by NIH grant R01 CA109560 and a seed grant from the Cancer League of Colorado to J.E. Slansky. R.H. McMahan and K.R. Jordan were supported in part by the Cancer Research Institute Predoctoral Emphasis Pathway in Tumor Immunology Fellowship. S.W. Dow was supported by NIH grant CA 86224-01. D.B. Wilson was supported by the Alzheimer's and Aging Research Center, the Osteoporosis and Breast Cancer Research Center, and NIH grant DA 015212.

Received for publication September 21, 2005, and accepted in revised form June 20, 2006.

Address correspondence to: Jill E. Slansky, Integrated Department of Immunology, University of Colorado at Denver and Health Sciences Center, 1400 Jackson Street, Room K511, Denver, Colorado 80206, USA. Phone: (303) 398-1887; Fax: (303) 398-1396; E-mail: Jill.Slansky@UCHSC.edu.
1. Dunn, G.P., Old, L.J., and Schreiber, R.D. 2004 The three Es of cancer immunoediting. Annu. Rev Immunol. 22:329-360.

2. Marincola, F.M., Jaffee, E.M., Hicklin, D.J., and Ferrone, S. 2000. Escape of human solid tumors from T-cell recognition: molecular mechanisms and functional significance. Adv. Immunol. 74:181-273.

3. Gorelik, L., and Flavell, R.A. 2001. Immune-mediated eradication of tumors through the blockade of transforming growth factor-beta signaling in T cells. Nat. Med. 7:1118-1122.

4. Niethammer, A.G., et al. 2002. A DNA vaccine against VEGF receptor 2 prevents effective angiogenesis and inhibits tumor growth. Nat. Med. 8:1369-1375

5. Novellino, L., Castelli, C., and Parmiani, G. 2005. A listing of human tumor antigens recognized by T cells: March 2004 update. Cancer Immunol. Immunother. 54:187-207.

6. Dyall, R, et al. 1998. Heteroclitic immunization induces tumor immunity. J. Exp. Med. 188:1553-1561.

7. Overwijk, W.W., et al. 1998. gp100/pmel 17 is a murine tumor rejection antigen: induction of "self"reactive, tumoricidal $\mathrm{T}$ cells using high-affinity, altered peptide ligand. J. Exp. Med. 188:277-286.

8. Valmori, D., et al. 1999. Optimal activation of tumor-reactive T cells by selected antigenic peptide analogues. Int. Immunol. 11:1971-1980.

9. Tangri, S., et al. 2001. Structural features of peptide analogs of human histocompatibility leukocyte antigen class I epitopes that are more potent and immunogenic than wild-type peptide. J. Exp. Med. 194:833-846.

10. Parkhurst, M.R., et al. 1996. Improved induction of melanoma-reactive CTL with peptides from the melanoma antigen gp100 modified at HLA-A*0201binding residues. J. Immunol. 157:2539-2548.

11. Hernandez, J., et al. 2004. Antigenicity and immunogenicity of peptide analogues of a low affinity peptide of the human telomerase reverse transcriptase tumor antigen. Eur. J. Immunol. 34:2331-2341.

12. Slansky, J.E., et al. 2000. Enhanced antigen-specific antitumor immunity with altered peptide ligands that stabilize the MHC-peptide-TCR complex. Immunity. 13:529-538.

13. de Visser, K.E., et al. 2001. Low-avidity self-specific $\mathrm{T}$ cells display a pronounced expansion defect that can be overcome by altered peptide ligands. J. Immunol. 167:3818-3828.

14. Hoffmann, T.K., et al. 2002. The ability of variant peptides to reverse the nonresponsiveness of T lymphocytes to the wild-type sequence p53(264-272) epitope. J. Immunol. 168:1338-1347.

15. Dudley, M.E., et al. 2002. Cancer regression and autoimmunity in patients after clonal repopulation with antitumor lymphocytes. Science. 298:850-854.

16. Anichini, A., et al. 1999. An expanded peripheral $\mathrm{T}$ cell population to a cytotoxic $\mathrm{T}$ lymphocyte (CTL)-defined, melanocyte-specific antigen in metastatic melanoma patients impacts on generation of peptide-specific CTLs but does not overcome tumor escape from immune surveillance in metastatic lesions. J. Exp. Med. 190:651-667.

17. Fong, L., et al. 2001. Altered peptide ligand vaccination with Flt3 ligand expanded dendritic cells for tumor immunotherapy. Proc. Natl. Acad. Sci. U. S. A. 98:8809-8814.

18. Skipper, J.C., et al. 1996. An HLA-A2-restricted tyrosinase antigen on melanoma cells results from posttranslational modification and suggests a novel pathway for processing of membrane proteins. J. Exp. Med. 183:527-534.

19. Rubio, V., et al. 2003. Ex vivo identification, isolation and analysis of tumor-cytolytic T cells. Nat. Med. 9:1377-1382.

20. Molldrem, J.J., et al. 2003. Chronic myelogenous leukemia shapes host immunity by selective deletion of high-avidity leukemia-specific T cells. J. Clin. 
Invest. 111:639-647. doi:10.1172/JCI200316398.

21. Stuge, T.B., et al. 2004. Diversity and recognition efficiency of T cell responses to cancer. PLoS Med. 1:e28.

22. Lee, P.P., et al. 1999. Characterization of circulating $T$ cells specific for tumor-associated antigens in melanoma patients. Nat. Med. 5:677-685.

23. Valitutti, S., Muller, S., Dessing, M., and Lanzavecchia, A. 1996. Different responses are elicited in cytotoxic T lymphocytes by different levels of T cell receptor occupancy. J. Exp. Med. 183:1917-1921.

24. Alexander-Miller, M.A., Leggatt, G.R., Sarin, A., and Berzofsky, J.A. 1996. Role of antigen, CD8, and cytotoxic T lymphocyte (CTL) avidity in high dose antigen induction of apoptosis of effector CTL. J. Exp. Med. 184:485-492.

25. Kalergis, A.M., et al. 2001. Efficient T cell activation requires an optimal dwell-time of interaction between the TCR and the PMHC complex. Nat. Immunol. 2:229-234.

26. Holler, P.D., and Kranz, D.M. 2003. Quantitative analysis of the contribution of TCR/pepMHC affinity and CD8 to $\mathrm{T}$ cell activation. Immunity. 18:255-264.

27. Rosette, C., et al. 2001. The impact of duration versus extent of TCR occupancy on T cell activation: a revision of the kinetic proofreading model. Immunity. 15:59-70.

28. Kersh, G.J., and Allen, P.M. 1996. Structural basis for $T$ cell recognition of altered peptide ligands: a single $T$ cell receptor can productively recognize a large continuum of related ligands. J. Exp. Med. 184:1259-1268.

29. Matsui, K., Boniface, J.J., Steffner, P., Reay, P.A., and Davis, M.M. 1994. Kinetics of T-cell receptor binding to peptide/I-Ek complexes: correlation of the dissociation rate with T-cell responsiveness. Proc. Natl. Acad. Sci. U. S. A. 91:12862-12866.

30. Yu, X.Z., Martin, P.J., and Anasetti, C. 2003. CD28 signal enhances apoptosis of CD8 T cells after strong TCR ligation. J. Immunol. 170:3002-3006.

31. Ueno, T., Tomiyama, H., Fujiwara, M., Oka, S., and Takiguchi, M. 2004. Functionally impaired HIVspecific CD8 T cells show high affinity TCR-ligand interactions. J. Immunol. 173:5451-5457.

32. Sykulev, Y., Vugmeyster, Y., Brunmark, A., Ploegh, H.L., and Eisen, H.N. 1998. Peptide antagonism and $\mathrm{T}$ cell receptor interactions with peptide-MHC complexes. Immunity. 9:475-483.

33. O'Connor, D.H., et al. 2002. Acute phase cytotoxic T lymphocyte escape is a hallmark of simian immunodeficiency virus infection. Nat. Med. 8:493-499.

34. Corbett, T.H., Griswold, D.P., Jr., Roberts, B.J., Peckham, J.C., and Schabel, F.M., Jr. 1977. Evaluation of single agents and combinations of chemotherapeutic agents in mouse colon carcinomas. Cancer. 40:2660-2680

35. Huang, A.Y., et al. 1996. The immunodominant major histocompatibility complex class I-restricted antigen of a murine colon tumor derives from an endogenous retroviral gene product. Proc. Natl.
Acad. Sci. U. S. A. 93:9730-9735.

36. Luznik, L., et al. 2003. Successful therapy of metastatic cancer using tumor vaccines in mixed allogeneic bone marrow chimeras. Blood. 101:1645-1652.

37. Rodolfo, M., et al. 1994. Cytotoxic T lymphocytes recognize tumor antigens of a murine colonic carcinoma by using different T-cell receptors. Int. J. Cancer. 57:440-447.

38. Nino-Vasquez, J.J., et al. 2004. A powerful combination: the use of positional scanning libraries and biometrical analysis to identify cross-reactive $\mathrm{T}$ cell epitopes. Mol. Immunol. 40:1063-1074.

39. O'Herrin, S.M., et al. 1997. Analysis of the expression of peptide-major histocompatibility complexes using high affinity soluble divalent $\mathrm{T}$ cell receptors. J. Exp. Med. 186:1333-1345.

40. Sharma, A.K., et al. 2001. Class I major histocompatibility complex anchor substitutions alter the conformation of T cell receptor contacts. J. Biol. Chem. 276:21443-21449.

41. Kersh, G.J., et al. 2001. Structural and functional consequences of altering a peptide $\mathrm{MHC}$ anchor residue. J. Immunol. 166:3345-3354.

42. al-Ramadi, B.K., Jelonek, M.T., Boyd, L.F., Margulies, D.H., and Bothwell, A.L. 1995. Lack of strict correlation of functional sensitization with the apparent affinity of $\mathrm{MHC} /$ peptide complexes for the TCR. J. Immunol. 155:662-673.

43. Fahmy, T.M., Bieler, J.G., Edidin, M., and Schneck, J.P. 2001. Increased TCR avidity after T cell activation: a mechanism for sensing low-density antigen. Immunity. 14:135-143.

44. Balendiran, G.K., et al. 1997. The three-dimensional structure of an H-2Ld-peptide complex explains the unique interaction of Ld with beta- 2 microglobulin and peptide. Proc. Natl. Acad. Sci. U. S. A. 94:6880-6885.

45. Speir, J.A., et al. 1998. Structural basis of 2C TCR allorecognition of $\mathrm{H}-2 \mathrm{Ld}$ peptide complexes. Immunity. 8:553-562.

46. Khilko, S.N., et al. 1993. Direct detection of major histocompatibility complex class I binding to antigenic peptides using surface plasmon resonance. Peptide immobilization and characterization of binding specificity. J. Biol. Chem. 268:15425-15434.

47. Dutoit, V., Guillaume, P., Cerottini, J.C., Romero, P., and Valmori, D. 2002. Dissecting TCR-MHC/ peptide complex interactions with A2/peptide multimers incorporating tumor antigen peptide variants: crucial role of interaction kinetics on functional outcomes. Eur. J. Immunol. 32:3285-3293.

48. Dow, S.W., et al. 1999. Lipid-DNA complexes induce potent activation of innate immune responses and antitumor activity when administered intravenously. I. Immunol. 163:1552-1561.

49. Zippelius, A., et al. 2004. Effector function of human tumor-specific CD8 $\mathrm{T}$ cells in melanoma lesions: a state of local functional tolerance. Cancer Res. 64:2865-2873.

50. Betts, M.R., et al. 2003. Sensitive and viable identi- fication of antigen-specific CD8+ T cells by a flow cytometric assay for degranulation. J. Immunol. Methods. 281:65-78.

51. Dighe, A.S., Richards, E., Old, L.J., and Schreiber, R.D. 1994. Enhanced in vivo growth and resistance to rejection of tumor cells expressing dominant negative IFN gamma receptors. Immunity. 1:447-456.

52. Robinson, R.A., and Lee, D.R. 1996. Studies of tumpeptide analogs define an alternative anchor that can be utilized by Ld ligands lacking the consensus P2 anchor. J. Immunol. 156:4266-4273.

53. Ikeda, H., Old, L.J., and Schreiber, R.D. 2002. The roles of IFN gamma in protection against tumor development and cancer immunoediting. Cytokine Growth Factor Rev. 13:95-109.

54. Anderton, S.M., Radu, C.G., Lowrey, P.A., Ward, E.S., and Wraith, D.C. 2001. Negative selection during the peripheral immune response to antigen. J. Exp. Med. 193:1-11.

55. Vezys, V., and Lefrancois, L. 2002. Cutting edge: inflammatory signals drive organ-specific autoimmunity to normally cross-tolerizing endogenous antigen. J. Immunol. 169:6677-6680.

56. Hernandez, J., Aung, S., Redmond, W.L., and Sherman, L.A. 2001. Phenotypic and functional analysis of CD8(+) T cells undergoing peripheral deletion in response to cross-presentation of self-antigen. J. Exp. Med. 194:707-717.

57. Huang, C.T., et al. 2003. CD4+ T cells pass through an effector phase during the process of in vivo tolerance induction. J. Immunol. 170:3945-3953.

58. Zajac, A.J., et al. 1998. Viral immune evasion due to persistence of activated $T$ cells without effector function. J. Exp. Med. 188:2205-2213.

59. Wherry, E.J., Blattman, J.N., Murali-Krishna, K., van der Most, R., and Ahmed, R. 2003. Viral persistence alters CD8 T-cell immunodominance and tissue distribution and results in distinct stages of functional impairment. J. Virol. 77:4911-4927.

60. Crawford, F., Kozono, H., White, J., Marrack, P., and Kappler, J. 1998. Detection of antigen-specific T cells with multivalent soluble class II MHC covalent peptide complexes. Immunity. 8:675-682.

61. Kappler, J., White, J., Kozono, H., Clements, J., and Marrack, P. 1994. Binding of a soluble alpha beta T-cell receptor to superantigen/major histocompatibility complex ligands. Proc. Natl. Acad. Sci. U. S. A. 91:8462-8466.

62. Templeton, N.S., et al. 1997. Improved DNA: liposome complexes for increased systemic delivery and gene expression. Nat. Biotechnol. 15:647-652.

63. Zaks, K., et al. 2006. Efficient immunization and cross-priming by vaccine adjuvants containing TLR3 or TLR9 agonists complexed to cationic liposomes. J. Immunol. 176:7335-7345.

64. Overwijk, W.W., Surman, D.R., Tsung, K., and Restifo, N.P. 1997. Identification of a Kb-restricted CTL epitope of beta-galactosidase: potential use in development of immunization protocols for "self" antigens. Methods. 12:117-123. 